\title{
Retraction Note to: Artifact Rejection for Concurrent TMS-EEG Data
}

\author{
Wei Wu, Corey Keller, and Amit Etkin
}

\section{Retraction Note to:}

Chapter 6 "Artifact Rejection for Concurrent TMS-EEG Data" in: Z. Chen, S.V. Sarma (eds.), Dynamic Neuroscience, https://doi.org/10.1007/978-3-319-71976-4_6

Chapter 6 (Artifact Rejection for Concurrent TMS-EEG Data) by Wei Wu, Corey Keller, and Amit Etkin, that was originally published in the volume has been removed by the request of the authors, as this work will be appearing in another publication. 